\title{
New Insights into SNR Evolution Revealed by the Discovery of Recombining Plasmas
}

\author{
Hiroya Yamaguchi \\ Harvard-Smithsonian Center for Astrophysics, 60 Garden Street, Cambridge, MA \\ 02138, USA \\ RIKEN (The Institute of Physical and Chemical Research), 2-1 Hirosawa, Wako, \\ Saitama 351-0198, Japan \\ Midori Ozawa and Takao Ohnishi \\ Kyoto University, Kitashirakawa-oiwake-cho, Sakyo, Kyoto 606-8502, Japan
}

\begin{abstract}
We report the discovery of recombining plasmas in three supernova remnants (SNRs) with the Suzaku X-ray astronomy satellite. During SNR's evolution, the expanding supernova ejecta and the ambient matter are compressed and heated by the reverse and forward shocks to form an X-ray emitting hot plasma. Since ionization proceeds slowly compared to shock heating, most young or middle-aged SNRs have ionizing (underionized) plasmas. Owing to high sensitivity of Suzaku, however, we have detected radiative recombination continua (RRCs) from the SNRs IC 443, W49B, and G359.1-0.5. The presence of the strong RRC is the definitive evidence that the plasma is recombining (overionized). As a possible origin of the overionization, an interaction between the ejecta and dense circumstellar matter is proposed; the highly ionized gas was made at the initial phase of the SNR evolution in dense regions, and subsequent rapid adiabatic expansion caused sudden cooling of the electrons. The analysis on the full X-ray band spectrum of IC 443, which is newly presented in this paper, provides a consistent picture with this scenario. We also comment on the implications from the fact that all the SNRs having recombining plasmas are correlated with the mixed-morphology class.
\end{abstract}

Key words: ISM: individual (IC 443, W49B, G359.1-0.5), Radiation mechanisms: thermal, Supernova remnants, X-rays: ISM

Email address: hyamaguchi@head.cfa.harvard.edu (Hiroya Yamaguchi). 


\section{Introduction}

Through a supernova (SN) explosion, huge energy (typically of the order of $10^{51} \mathrm{erg}$ ) is released in form of the kinetic energy of the ejecta. Since the supernova remnant (SNR) expands supersonically, shock waves are formed to compress and heat the ejecta and ambient matter. The shocks generally propagate in an extremely low-density $\left(\lesssim 1 \mathrm{~cm}^{-3}\right)$ interstellar medium (ISM) and hence are nearly collisionless; the shock transition occurs on a length scale much shorter than the typical particle mean free path to Coulomb collisions. Therefore, in the early phases of the SNR evolution, the shocked plasma is expected to be far from equilibrium in terms of the particle (electron and ion) temperatures. Furthermore, due to the small cross sections for the ionization and recombination processes, non-equilibrium ionization (NEI) is expected in most SNRs. The ionization timescale, a key parameter for the NEI state, is defined as $n_{e} t$, the product of the electron density and the time since the gas was heated. Typically, $n_{e} t$ is required to be $\gtrsim 10^{12} \mathrm{~cm}^{-3} \mathrm{~s}$ for collisional ionization equilibrium (CIE) (e.g., Masai 1984). It has been reported that most young or middle-aged SNRs in our Galaxy (e.g., Tycho: Hwang et al. 1998; SN 1006: Yamaguchi et al. 2008; Cygnus Loop: Miyata et al. 2007) or the Large Magellanic Cloud (e.g., Hughes et al. 1998) exhibit an ionizing (underionized) NEI plasma with $n_{e} t<10^{12} \mathrm{~cm}^{-3} \mathrm{~s}$, where the rate of the ionization process always exceeds the rate of the recombination process.

In contrast, ASCA observations of the SNRs IC 443 and W49B revealed that the ionization degrees (or ionization temperature 1 , $k T_{z}$ ) of some heavy elements were significantly higher than those expected from the electron temperature $k T_{e}$ (Kawasaki et al. 2002; 2005). This suggests that the plasmas are recombining (overionized), which is quite unusual for the thermal evolution of SNRs evolving in tenuous ISM. However, following XMM-Newton observations showed that these SNRs' spectra can be represented by a model of a CIE plasma, and the overionization is only marginal (Troja et al. 2008; Miceli et al. 2006). The presence of recombining plasmas in SNRs has, therefore, been a subject of debate.

1 The ionization temperature is defined to represent the degree of ionization for each individual element. When the average charge of the atoms is consistent with that in a CIE plasma with an electron temperature of $k T_{e}^{\prime}, k T_{z}$ is given to equal $k T_{e}^{\prime}$. 

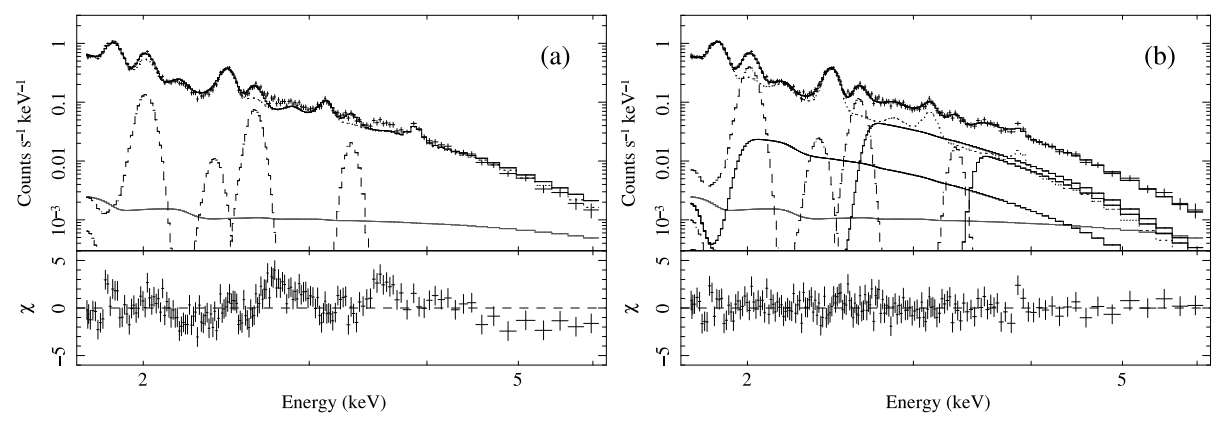

Fig. 1. (a) XIS spectrum of IC 443 in the $1.75-6.0 \mathrm{keV}$ band, fitted with the CIE plasma (black dotted line), additional Lyman lines (black dashed lines; see text), and cosmic X-ray background (gray solid line). The lower panel shows the residual from the best-fit model. Two broad features are seen at $\sim 2.7 \mathrm{keV}$ and $\sim 3.5 \mathrm{keV}$. (b) Same spectrum as (a), but for a fit with RRC components of $\mathrm{H}$-like $\mathrm{Mg}$, Si, and $\mathrm{S}$ (black solid lines). The residuals seen in (a) are now gone.

\section{Recent Suzaku discovery of recombining plasmas}

The discoveries of recombination continua (RRCs) from several SNRs were recent breakthroughs made by Suzaku's high sensitivity to extended sources, because the presence of the strong $\mathrm{RRC}$ is definitive evidence for the recombining plasma. Here we review the results of spectroscopic studies of IC 443 (\$2.1), W49B (\$2.2), and G359.1-0.5 (\$2.3) using X-ray Imaging Spectrometer (XIS) on board Suzaku. Details for the observations and analysis procedure are found in Yamaguchi et al. (2009), Ozawa et al. (2009), and Ohnishi et al. (2011), for IC 443, W49B, and G359.1-0.5, respectively.

\section{$2.1 \quad I C 443$}

IC 443 (the Jellyfish Nebula) is located near the Gem OB1 association and a dense giant molecular cloud (Cornett et al. 1977), suggesting that the remnant originated from a collapse of a massive star. The SNR likely evolved in a low density cavity formed by the progenitor's stellar wind activity and recently encountered the cavity wall or the molecular cloud (Braun \& Strom 1986; Troja et al. 2006; 2008). The age of the SNR was estimated to be $\sim 4000 \mathrm{yr}$ from the radii of the forward and reverse shocks (Troja et al. 2008).

The first claim of overionization in this SNR is presented by the ASCA observation (Kawasaki et al. 2002). They measured intensity ratios of the Hlike to He-like $\mathrm{K} \alpha$ lines of $\mathrm{S}$ to obtain $k T_{z}=1.5 \mathrm{keV}$ for this element. This value is significantly higher than the electron temperature of $1.0 \mathrm{keV}$ measured from the slope of the bremsstrahlung continuum spectrum. They also found the excess of the intensity in the Si Ly $\alpha$ line over that expected from a $1.0 \mathrm{keV}$ 
CIE plasma, implying the overionization of Si as well. However, they were not aware of presence of RRC emissions which are expected to be strong in an overionized plasma with the derived temperatures.

Figure 1 shows the XIS spectrum in the energies above the $\mathrm{Si}-\mathrm{K} \alpha$ line extracted from a representative region of the SNR. The residuals to a spectral model consisting of a CIE plasma (an APEC model: Smith et al. 2001) show distinct excess Lyman emission from the H-like ions of Si, S, and Ar, as already reported by Kawasaki et al. (2002). After adding Gaussian components to account for these features, we found significant hump-like residuals around $\sim 2.7 \mathrm{keV}$ and $\sim 3.5 \mathrm{keV}$ (Figure 1 a). These do not correspond to emission lines from any abundant element, but are highly consistent with the K-shell binding potentials $\left(I_{z}\right)$ of the H-like $\mathrm{Si}(2666 \mathrm{eV})$ and $\mathrm{S}(3482 \mathrm{eV})$. Therefore, the residuals likely arise from free-bound transition to the K-shells of the H-like Si and S.

When the electron temperature is much lower than the K-edge energy $\left(k T_{e} \ll I_{z}\right)$, a formula for an RRC spectrum is approximated as

$$
\frac{d P}{d E}\left(E_{\gamma}\right) \propto \exp \left(-\frac{E_{\gamma}-I_{z}}{k T_{e}}\right), \text { for } E_{\gamma} \geq I_{z}
$$

After adding the RRC components, the fit was dramatically improved, as shown in Figure1 b. The electron temperature was derived to be $k T_{e} \sim 0.6 \mathrm{keV}$. We found that the flux ratios of the $\mathrm{H}$-like RRC to the He-like $\mathrm{K} \alpha$ lines correspond to the ionization temperatures of $k T_{z} \sim 1.0 \mathrm{keV}$ and $k T_{z} \sim 1.2 \mathrm{keV}$ for $\mathrm{Si}$ and $\mathrm{S}$, respectively. These values are significantly higher than the electron temperature, indicating directly that the plasma is overionized.

\section{2 $W 49 B$}

W49B is likely to be associated with the star forming region W49A (Brogan \& Troland 2001). The SNR exhibits centrally filled X-rays with a bright radio shell. The near-infrared image shows a barrel-shaped structure with coaxial rings, which is suggestive of bipolar stellar wind structures surrounding a massive progenitor (Keohane et al. 2007). Therefore, W49B had likely exploded inside a wind-blown bubble. The age of the SNR is uncertain, but is roughly estimated to be 1000 yr (e.g., Lopez et al. 2009).

Using ASCA data, Kawasaki et al. (2005) derived the ionization temperatures of $\mathrm{Ar}$ and $\mathrm{Ca}$ to be $k T_{z} \sim 2.5 \mathrm{keV}$ from intensity ratios of the H-like to He-like $\mathrm{K} \alpha$ emissions. However, a similar diagnostics was not performed for Fe, possibly due to the limited photon statistics. It should also be noted that 


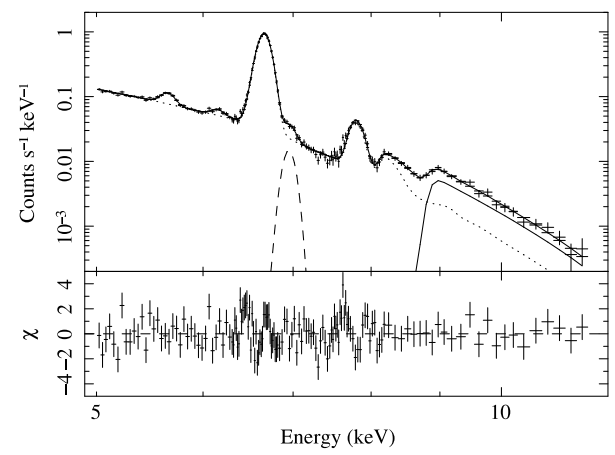

Fig. 2. XIS spectrum of W49B in the $5.0-12 \mathrm{keV}$ band. The solid, dotted, and dashed lines represent the RRC of He-like Fe, CIE plasma, and additional Gaussian accounting for the strong $\operatorname{Ly} \alpha$ line, respectively.

emission lines of $\mathrm{Cr}$ and $\mathrm{Mn}$ were detected in the ASCA spectrum (Hwang et al. 2000). This is the first detection of these elements in a celestial X-ray source.

Figure 2 shows the XIS spectrum above $5 \mathrm{keV}$ extracted from the entire SNR. The spectrum exhibits K $\alpha$ emission lines of $\mathrm{Cr}, \mathrm{Mn}, \mathrm{Fe}$, and Ni (blended with Fe-K $\beta$ ). In addition, we can see a spectral hump at $\sim 9 \mathrm{keV}$. This structure cannot be explained by any combination of high temperature plasmas either in the CIE or underionized NEI state, but is likely to be caused by an RRC of He-like Fe $\left(I_{z}=8830 \mathrm{eV}\right)$. In fact, addition of the RRC, together with the Fe-Ly $\alpha$ line, to the CIE plasma component greatly improved the spectral fit. The electron and ionization temperatures we derived are $k T_{e} \sim 1.5 \mathrm{keV}$, and $k T_{z} \sim 2.7 \mathrm{keV}$, respectively. Although we did not detect RRC features of lower- $Z$ elements, we confirmed that the H-like to He-like line ratios of $\mathrm{Ar}$ and $\mathrm{Ca}$ are significantly larger than those expected for a $1.5 \mathrm{keV}$ CIE plasma, as was firstly claimed by Kawasaki et al. (2005). This result will be reported in detail in a future paper.

Following our result, Miceli et al. (2010) confirmed the presence of the strong RRC using XMM-Newton data. Utilizing the higher angular resolution, they also investigated the spatial distribution of the recombining plasma and found that the highly overionized matters are localized at the center of the SNR.

\section{$2.3 \quad G 359.1-0.5$}

G359.1-0.5, an SNR located in the direction of the Galactic center, was discovered by a $4.9 \mathrm{GHz}$ radio observation (Downes et al. 1979). The angular size of $\sim 20^{\prime}$ corresponds to the diameter of $\sim 50 \mathrm{pc}$ (at a distance of $8.5 \mathrm{kpc}$ ). This large value suggests that G359.1-0.5 is more evolved than IC 443 and W49B. The SNR is likely to be interacting with surrounding molecular clouds, 


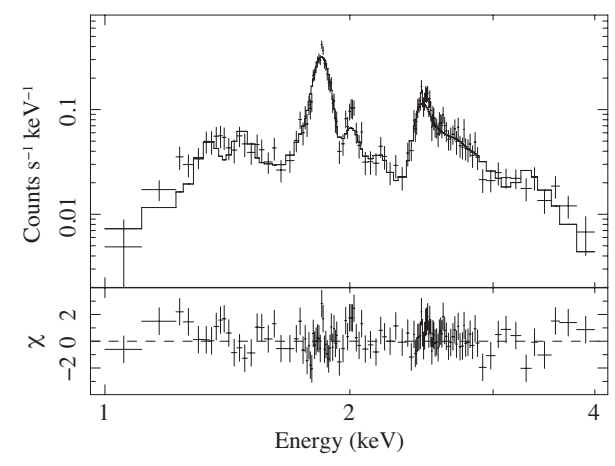

Fig. 3. XIS spectrum of G359.1-0.5, fitted with an overionized plasma model.

since bright emissions of $1720 \mathrm{MHz} \mathrm{OH}$ maser and shocked molecular hydrogen had been detected at the SNR's limb (Yusef-Zadeh et al. 1995; Lazendic et al. 2002).

Using ASCA, Bamba et al. (2000) revealed that the X-ray spectrum exhibits prominent emission lines of $\mathrm{Si}$ and $\mathrm{S}$. They also claimed that the centroid energies of the $\mathrm{Si}$ - and $\mathrm{S}-\mathrm{K} \alpha$ lines are consistent with those of He-like $\mathrm{Si}$ and H-like S ( Ly $\alpha)$, respectively. This is, if true, quite peculiar because the heavier elements tend to be less ionized compared to the lighter ones in a normal isothermal plasma. In fact, the model they used to reproduce the observed spectrum was composed of two rather non-physical plasma components: a cooler one consisting only of $\mathrm{Si}$, and a hotter one with extremely over abundant S. In the ASCA spectrum, however, the He-like and H-like lines were not distinctly separated due to the limited photon statistics and the degraded energy resolution.

The recent Suzaku observation has provided an interesting result which unveiled the nature of this peculiar spectrum. The XIS spectrum of the entire $\mathrm{SNR}$ is shown in Figure 3. In addition to the $\mathrm{K} \alpha$ emission lines of He-like Si/S and $\mathrm{H}$-like $\mathrm{Si}$, we can see a broad shoulder-like structure above the energy of the He-like $\mathrm{S}-\mathrm{K} \alpha$ line $(\sim 2.4 \mathrm{keV})$. This structure is analogous to the RRC of Si observed in IC 443. We thus introduced a recombining plasma model provided by the SPEX software package (Kaastra et al. 1996). The best-fit electron and ionization temperatures were obtained to be $k T_{e} \sim 0.3 \mathrm{keV}$ and $k T_{z} \sim$ $0.8 \mathrm{keV}$, respectively. The recombining state is, therefore, clearly indicated. The shoulder-like spectral feature was confirmed to be the RRC of He-like Si $\left(I_{z}=2439 \mathrm{eV}\right)$. In the previous study by Bamba et al. (2000), this feature was misinterpreted as a S-Ly $\alpha$ line and hence presence of an unreasonably-hightemperature component was claimed. We also attempted to fit the spectrum with a two-component CIE plasma model as was performed by Bamba et al. (2000), but the model failed to reproduce the overall spectrum. 

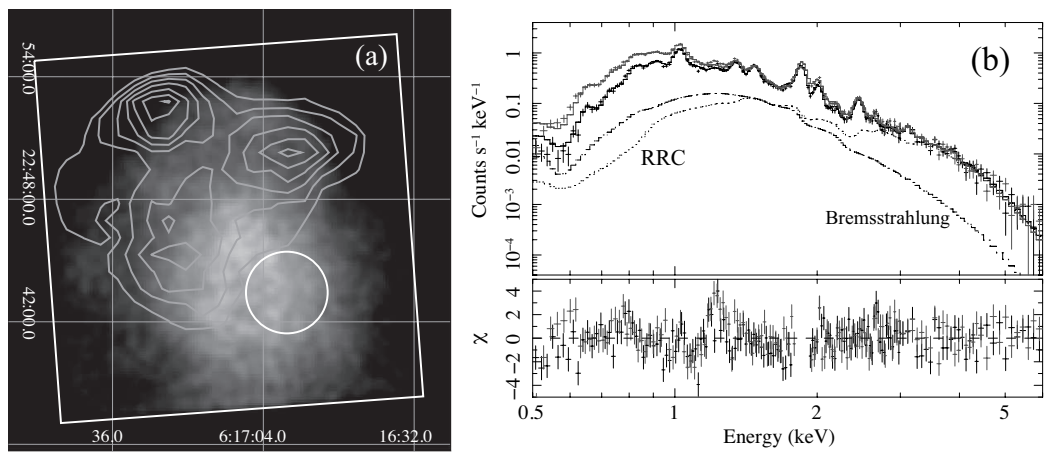

Fig. 4. (a) XIS image of the northern part of IC 443 in the $1.7-3.0 \mathrm{keV}$ band (gray scale), overlaid with contours of the $0.6-1.0 \mathrm{keV}$ emission. The coordinates of (R.A. and decl.) refer to epoch J2000.0. The white square and circle are the XIS field of view and the region where the spectrum is extracted, respectively. (b) Full-band XIS spectrum of IC 443, where the background is subtracted. Black and gray represent the data from the XIS0 and XIS1, respectively. (The XIS3 data is also analyzed simultaneously, but is not shown for simplicity.) The contributions from the bremsstrahlung and RRCs (for the XIS0) are individually indicated.

\section{$3 \quad$ Full X-ray band spectrum of IC 443}

In this section, we present our new result of the spectral analysis on IC 443 using a recombining plasma model. The analyzed energy range is extended to include the soft X-ray band as well. Figure 4 a shows the $1.7-3.0 \mathrm{keV}$ (hard) image of the northern part of IC 443 (Yamaguchi et al. 2009), overlaid with contours of the $0.6-1.0 \mathrm{keV}$ (soft) band. The shell-like structure seen in the soft X-ray image originates mainly from the surrounding clouds which are recently shocked by the blast wave (Kawasaki et al. 2002; Troja et al. 2006). To avoid contamination from this emission component as far as possible, we extract XIS spectra from a narrow circular region with a radius of 2 arcmin shown in Figure 4a. The background was taken from the "ANTI GALACTIC" field (Masui et al. 2009) whose aim point was $(l, b)=(235.0,0.0)$. To minimize the uncertainty due to the background subtraction, in particular that of the particle component (non X-ray background), we extracted the background spectra from the same detector coordinates as the source regions. The background-subtracted spectra are shown in Figure 4b.

As mentioned in Section 2.1, the ionization temperatures of $\mathrm{Si}$ and $\mathrm{S}$ are different from each other $(1.0 \mathrm{keV}$ and $1.2 \mathrm{keV}$, respectively). This implies that the single- $k T_{z}$ model, which was applied for G359.1-0.5, does not work in this case. Therefore, we used an NEI model of SPEX, where the free parameters are the initial plasma temperature $\left(k T_{[\text {init] }}\right)$, post-shock electron temperature $\left(k T_{e}\right)$, ionization timescale $\left(n_{e} t\right)$, emission measure $\left(n_{e} n_{\mathrm{H}} V\right)$, abundances of different elements, and absorption column $\left(N_{\mathrm{H}}\right)$. The first parameter is usually set to a very low value $(\sim 1 \mathrm{eV}$, for instance) to describe a standard under- 
Table 1

Best-fit spectral parameters for IC 443.

\begin{tabular}{lc}
\hline Parameters & Values \\
\hline$N_{\mathrm{H}}\left(10^{21} \mathrm{~cm}^{-2}\right)$ & $5.2 \pm 0.1$ \\
$k T_{[\mathrm{init}]}(\mathrm{keV})$ & $2.8_{-0.5}^{+0.8}$ \\
$k T_{e}(\mathrm{keV})$ & $0.58 \pm 0.07$ \\
$n_{e} t\left(10^{11} \mathrm{~cm}^{-3} \mathrm{~s}\right)$ & $6.7 \pm 0.5$ \\
$\mathrm{Ne}, \mathrm{Na}($ solar $)$ & $0.83 \pm 0.04$ \\
$\mathrm{Mg}, \mathrm{Al}($ solar $)$ & $0.69_{-0.04}^{+0.05}$ \\
$\mathrm{Si}($ solar $)$ & $1.2 \pm 0.1$ \\
$\mathrm{~S}($ solar $)$ & $1.1 \pm 0.1$ \\
$\mathrm{Ar}, \mathrm{Ca}($ solar $)$ & $1.3 \pm 0.3$ \\
$\mathrm{Fe}, \mathrm{Ni}($ solar $)$ & $0.30 \pm 0.01$ \\
$n_{e} n_{\mathrm{H}} V\left(10^{56} \mathrm{~cm}^{-3}\right)$ & $8.2 \pm 0.3$ \\
\hline$\chi^{2} /$ dof & $722 / 430$ \\
\hline
\end{tabular}

Statistic errors are for $1 \sigma$ confidence.

ionized NEI plasma, but in our case it should be allowed to vary freely and also to be higher than the $k T_{e}$ value for reproducing an overionized plasma. 2 Since the gain calibration of the XIS is reported to be problematic around the energy of the Si K-edge (1.84 keV), we ignore the energy range of 1.8-1.9 keV from the fitting. The best-fit parameters obtained with this model is given in Table 1. Although the fit is unacceptable from a statistical point of view $\left(\chi^{2} /\right.$ dof $\left.=722 / 430\right) 3$ the overall spectrum was well reproduced by the model consisting only of one plasma component. It is worth noting that an X-ray spectrum from similarly narrow regions in IC 443 was modeled with at least two components of CIE and/or underionized plasmas with different electron temperatures in all the previous works (e.g., Troja et al. 2008; Bocchino et al. 2009). Introducing the recombining plasma model, however, we found that multiple temperatures are not always needed. This is because the strong RRCs contribute largely to the continuum flux in the hard X-ray band, even though the slope of the bremsstrahlung emission is steep due to the low $(\sim 0.6 \mathrm{keV})$ electron temperature (see Figure 4b).

$\overline{2}$ More detailed information about this model is found at the URL $<$ http://www.sron.nl/files/HEA/SPEX/manuals/manual.pdf $>$

3 The largest discrepancy between the data and model is seen around $1.2 \mathrm{keV}$, where the Fe L-shell emissivity is known to be highly uncertain (e.g., Kosenko et al. 2008). 
Assuming the plasma depth of $3 \mathrm{pc}$, the emitting volume is estimated to be $2.2 \times 10^{56} \mathrm{~cm}^{3}$. The emission measure is, therefore, converted to the uniform electron density of $2.0 \mathrm{~cm}^{-3}$. The enhanced abundance ratios of $\mathrm{Si} / \mathrm{Fe}$ and $\mathrm{S} / \mathrm{Fe}$ with respect to the solar values are typical for core-collapse SNRs, but the Fe abundance would be relatively uncertain due to the systematic uncertainty in the Fe L-shell emissivity.

\section{Discussion}

\subsection{Origin of the recombining plasmas}

Utilizing the high spectral sensitivity of Suzaku, we have discovered recombining (overionized) plasmas in the three middle-aged SNRs, IC 443, W49B, and G359.1-0.5, for the first time. To date, astronomical recombining plasmas had been observed in relatively compact regions, such as X-ray binaries (e.g., Cygnus X-3: Kawashima \& Kitamoto 1996) or planetary nebulae (e.g., BD $+30^{\circ} 3639$ : Nordon et al. 2009), but those in extended sources had not been reported. As argued in Section 1, SNRs usually have ionizing plasmas as the shock-heated plasma slowly achieves CIE. Therefore, the discoveries of the recombining plasmas are dramatically changing our understanding of SNR dynamics and evolution.

Recombining plasma can be produced by strong radiation from an external X-ray source (photoionization). In IC 443, there is an X-ray pulsar at the center of the remnant (CXOU J061705.3+222127: Olbert et al. 2001). However, the X-ray luminosity of this source is too faint to produce the ionization balance we observed, as already discussed by Kawasaki et al. (2002). No central source has been found in W49B and G359.1-0.5. Early photoionization by X-ray bursts (i.e., X-ray flash and/or Gamma-ray burst afterglow) occurred immediately after the SN explosion is also unlikely, because this process affects only surrounding ISM and hence cannot explain our results; the SN ejecta are highly overionized (Ozawa et al. 2009; Ohnishi et al. 2011).

As the mechanism to form the recombining plasma, we here propose rapid adiabatic cooling occurred due to fast expansion of SNRs as discussed by Itoh \& Masai (1989). If an SN explodes in dense circumstellar matter (CSM) formed by the progenitor's stellar wind activity, both CSM and SN ejecta can be shock-heated to high temperature at the initial phase of the SNR's evolution. Furthermore, the plasmas can be in almost CIE as they have large electron densities. Such an CSM/ejecta interaction and a resultant high-temperature CIE plasma were observed in SN 1993J at a few days after the explosion (Kohmura et al. 1994; Uno et al. 2002). Although the shock velocity is signif- 
Table 2

Summary of the observed parameters and estimations of timescale to reach CIE.

\begin{tabular}{lccccc}
\hline & $\begin{array}{c}k T_{e} \\
(\mathrm{keV})\end{array}$ & $\begin{array}{c}n_{e} \\
\left(\mathrm{~cm}^{-3}\right)\end{array}$ & $\begin{array}{c}\text { Overionized } \\
\text { elements }\end{array}$ & $\begin{array}{c}t_{\mathrm{CIE}} \\
(\mathrm{yr})\end{array}$ & $\begin{array}{c}\text { Age } \\
(\mathrm{yr})\end{array}$ \\
\hline IC443 & 0.6 & 2.0 & $\mathrm{Si}, \mathrm{S}$ & $\sim 2 \times 10^{4}$ & $\sim 4 \times 10^{3 *}$ \\
W49B & 1.5 & 2.0 & $\mathrm{Fe},(\mathrm{Ar}, \mathrm{Ca})^{\ddagger}$ & $\sim 1 \times 10^{4}$ & $\sim 1 \times 10^{3 \dagger}$ \\
G359.1-0.5 & 0.3 & 0.2 & $\mathrm{Si}, \mathrm{S}$ & $\sim 1 \times 10^{4}$ & Unknown \\
\hline
\end{tabular}

${ }^{*}$ Troja et al. (2008); ${ }^{\dagger}$ Lopez et al. (2009); ${ }^{\ddagger}$ Claimed by Kawasaki et al. (2005).

icantly decreased by the dense matter, it can quickly recover when the blast wave penetrates into the outer ISM region (Itoh \& Masai 1989; a similar suggestion is given by Dwarkadas (2005), but for shocks passing through more expanded wind-blown shells). If the shocked electrons cool adiabatically much faster than the recombination timescale of the ionized materials, overionized plasma can be formed and survive for a long time.

This picture is supported by our new result on IC 443 where the overionized NEI model is applied. The best-fit parameters we obtained (Table 1) can be interpreted with the scenario that the $2.8 \mathrm{keV}\left(k T_{[\mathrm{init}]}\right)$ plasma in almost CIE suddenly cooled down to $0.6 \mathrm{keV}\left(k T_{e}\right)$, in the initial evolution phase, to form the recombining state. From the ionization timescale of $6.7 \times 10^{11} \mathrm{~cm}^{-3} \mathrm{~s}$, we estimate the time since the sudden cooling to be $t=1.1 \times 10^{4}\left(n_{e} / 2.0 \mathrm{~cm}^{-3}\right)^{-1} \mathrm{yr}$. Although the value is about three times larger than the known age of the remnant (4000 yr; Troja et al. 2008), this is not surprising because the actual evolution must be more complex; both the electron density and temperature are time-dependent. Future works taking into account the realistic evolutions are, therefore, highly encouraged to examine this scenario more precisely.

It should also be noted that an alternative scenario to create the recombining plasma has recently been proposed by Zhou et al. (2011) as an application of hydrodynamic simulation to W49B. They found that adiabatic expansion causes rapid cooling of the ejecta (as was argued by Itoh \& Masai 1989) but thermal conduction occurring in a mixture of ambient clouds and a hot plasma plays an important role to form the recombining plasma. This scenario successfully explains the observational result from XMM-Newton (Miceli et al. 2010); the strong overionization is localized at the SNR's center whereas no overionized stuff is present in the eastern region where the ejecta expansion is hampered by the molecular cloud.

In Table 2, we summarize the physical parameters of the three SNRs with estimates of the recombination timescales (to reach $90 \%$ of CIE) using the calculation by Smith \& Hughes (2010). In IC 443 and W49B, the timescales are significantly larger than the ages of the remnants. Therefore, both the scenarios 
of the rapid adiabatic expansion and thermal conduction are supported. The age of G359.1-0.5 is currently unknown, but it should be less than about $1 \times 10^{4} \mathrm{yr}$ if the former scenario is the case.

\subsection{Relation with mixed-morphology class}

It is notable that all the three SNRs that have shown the recombining plasmas are classified as mixed-morphology (MM) SNRs (Rho \& Petre 1998). The MM-SNRs are characterized by a centrally-peaked X-ray profile with a more limb-brightened radio morphology. The X-ray emission is thermal in nature (in contrast to Crab-like pulsar wind nebulae), and generally exhibits a relatively uniform radial temperature profile (e.g., Slane et al. 2002). These properties are in distinct contrast to the expected properties of evolved SNRs in a homogeneous ISM; the Sedov solution predicts a limb-brightened X-ray morphology and a temperature profile declining steeply from the SNR center to the limb. Therefore, more complex theoretical models invoking thermal conduction (e.g., Shelton et al. 1999) or cloud evaporation (e.g., White \& Long 1991) have been proposed to explain a mechanism responsible for the observed properties of MM-SNRs. However, application of these models to individual SNRs has been largely unsuccessful to date (e.g., Slane et al. 2002; Bocchino et al. 2009), suggesting that the situation is more complex.

So far, all the theoretical models for MM-SNRs have assumed that the plasmas are always in CIE. Our new results, however, require a modified picture and strongly constrain any model that can provide a significant amount of overionized materials. As already mentioned, Itoh \& Masai (1989) predicted that the recombining plasma can be created if an SNR initially evolves in dense CSM produced by progenitor's stellar winds. Interestingly, it is suggested that density gradients in pre-existing CSM could be a major contributor to the emission profiles in the MM-SNRs (Petruk 2001). All the SNRs we have presented are associated with the massive star forming regions (and thus possibly with Type II progenitors), supporting the presence in the past of stellar wind materials. Future efforts for modeling the MM-SNRs, therefore, should properly consider the effects of ISM nonuniformity to both the dynamical and ionization evolutions in the SNRs. This will help improve our knowledge about the poorly understood MM-SNRs as well as unusual overionized plasmas in this class. 


\section{Conclusion}

We have discovered the strong RRC emissions from the three MM-SNRs, IC 443, W49B, and G359.1-0.5, for the first time. Since plasmas heated by forward or reverse shocks are expected to be ionizing (underionized) in usual SNRs, this discovery is dramatically changing our understanding of SNR dynamics and evolution. We have also demonstrated that a one-component overionized plasma model can reasonably reproduce the full X-ray band spectrum of IC 443 that has so far been modeled with at least two components of CIE and/or underionized NEI plasmas. As the origin of the overionization, rapid adiabatic cooling after the interaction between the pre-exiting CSM and SN ejecta is proposed. Alternatively, thermal conduction may work effectively to form the recombining plasmas in some cases. Future theoretical works properly taking into account the ISM nonuniformity and ionization nonequilibrium will help reveal the detailed mechanisms to form the overionization and centrallyfilled X-ray morphology in these remnants.

The authors appreciate a number of constructive suggestions from the anonymous referees. We also thank to Drs. Katsuji Koyama, Kuniaki Masai, Randall K. Smith, Patrick O. Slane, and Jelle Kaastra for helpful discussion. H.Y. is supported by Japan Society for the Promotion of Science (JSPS) Research Fellowship for Research Abroad.

\section{References}

Bamba, A., Yokogawa, J., Sakano, M., Koyama, K. Deep X-Ray Observations of Supernova Remnants G359.1-0.5 and G359.0-0.9 with ASCA, Publications of the Astronomical Society of Japan, 52, 259-266, 2000

Bocchino, F., Miceli, M., Troja, E. On the metal abundances inside mixedmorphology supernova remnants: the case of IC 443 and G166.0+4.3, Astronomy and Astrophysics, 498, 139-145, 2009

Braun, R., Strom, R. G. The structure and dynamics of evolved supernova remnants - The IC 443 complex, Astronomy and Astrophysics, 164, 193207, 1986

Brogan, C. L., Troland, T. H. Very Large Array H I Zeeman Observations toward the W49 Complex, The Astrophysical Journal 550, 799-816, 2001

Cornett, R. H., Chin, G., Knapp, G. R. Observations of CO emission from a dense cloud associated with the supernova remnant IC 443, Astronomy and Astrophysics, 54, 889-894, 1977

Downes, D., Goss, W. M.; Schwarz, U. J., Wouterloot, J. G. A. Compact radio sources in the galactic center region, Astronomy and Astrophysics Supplement Series, 35, 1-22, 1979 
Dwarkadas, V. V. The Evolution of Supernovae in Circumstellar Wind-Blown Bubbles. I. Introduction and One-Dimensional Calculations, The Astrophysical Journal 630, 892-910, 2005

Hughes, J. P., Hayashi, I., Koyama, K. ASCA X-Ray Spectroscopy of Large Magellanic Cloud Supernova Remnants and the Metal Abundances of the Large Magellanic Cloud The Astrophysical Journal 505, 732-748, 1998

Hwang, U., Hughes, J. P., Petre, R. The X-Ray Iron Emission from Tycho's Supernova Remnant, The Astrophysical Journal, 497, 833-841, 1998

Hwang, U., Petre, R., Hughes, J. P. The X-Ray Line Emission from the Supernova Remnant W49B, The Astrophysical Journal, 532, 970-979, 2000

Itoh, H., Masai, K. The effect of a circumstellar medium on the X-ray emission of young remnants of Type II supernovae, Royal Astronomical Society, Monthly Notices, 236, 885-899, 1989

Kaastra, J. S., Mewe, R., Nieuwenhuijzen, H. UVand X-ray Spectroscopy of Astrophysical and LaboratoryPlasmas, 411, 1996

Kawasaki, M. T., Ozaki, M., Nagase, F., et al. ASCA Observations of the Supernova Remnant IC 443: Thermal Structure and Detection of Overionized Plasma, The Astrophysical Journal, 572, 897-905, 2002

Kawasaki, M. T., Ozaki, M., Nagase, F., et al. Ionization States and Plasma Structures of Mixed-Morphology Supernova Remnants Observed with ASCA, The Astrophysical Journal, 631, 935-946, 2005

Kawashima, K, Kitamoto, S. Photoionized Plasma in Cygnus X-3, Publications of the Astronomical Society of Japan, 48, L113-L116, 1996

Keohane, J. W., Reach, W. T., Rho, J., Jarrett, T. H. A Near-Infrared and XRay Study of W49 B: A Wind Cavity Explosion, The Astrophysical Journal, 654, 938-944, 2007

Kohmura, Y., Inoue, H., Aoki, T., et al. Detection of X-rays from SN 1993J with ASCA, Publications of the Astronomical Society of Japan, 46, L157L161, 1994

Kosenko, D., Vink, J., Blinnikov., S., and Rasmussen, A. XMM-Newton Xray spectra of the SNR 0509-67.5: data and models, Astronomy and Astrophysics, 490, 223-230, 2008

Lazendic, J. S., Wardle, M., Burton, M. G. Shocked molecular gas towards the supernova remnant G359.1-0.5 and the Snake, Monthly Notices of the Royal Astronomical Society, 331, 537-544, 2002

Lopez, L. A., Ramirez-Ruiz, E., Pooley, D. A., Jeltema, T. E. Tools for Dissecting Supernova Remnants Observed with Chandra: Methods and Application to the Galactic Remnant W49B, The Astrophysical Journal, 691, 875-893, 2009

Masai, K. X-ray emission spectra from ionizing plasmas, Astrophysics and Space Science, 98, 367-395, 1984

Masui, K., Mitsuda, K., Yamasaki, N., et al. The Nature of Unresolved Soft X-Ray Emission from the Galactic Disk, Publications of the Astronomical Society of Japan, 61, S115-S122, 2009

Miceli, M., Bocchino, F., Decourchelle, A., et al. Spatial identification of the 
overionized plasma in W49B, Astronomy and Astrophysics, 514, L2-L5, 2010

Miceli, M., Decourchelle, A., Ballet, J., et al. The X-ray emission of the supernova remnant W49B observed with XMM-Newton, Astronomy and Astrophysics, 453, 567-578, 2006

Miyata, E., Katsudam S., Tsunemi, H., et al. Detection of Highly-Ionized Carbon and Nitrogen Emission Lines from the Cygnus Loop Supernova Remnant with the Suzaku Observatory, Publications of the Astronomical Society of Japan, 59, S163-S170, 2007

Nordon, R., Behar, E., Soker, N., et al. Narrow Radiative Recombination Continua: A Signature of Ions Crossing the Contact Discontinuity of Astrophysical Shocks, The Astrophysical Journal, 695, 834-843, 2009

Ohnishi, T., Koyama, K., Tsuru, T. G., et al. X-Ray Spectrum of a Peculiar Supernova Remnant, G359.1-0.5, Publications of the Astronomical Society of Japan, 63, 527-533, 2011

Olbert, C. M., Clearfield, C. R., Williams, N. E., et al. A Bow Shock Nebula Around a Compact X-Ray Source in the Supernova Remnant IC 443, The Astrophysical Journal, 554, L205-L208, 2001

Ozawa, M., Koyama, K., Yamaguchi, H., et al. Suzaku Discovery of the Strong Radiative Recombination Continuum of Iron from the Supernova Remnant W49B, The Astrophysical Journal, 706, L71-L75, 2009

Petruk, O. Thermal X-ray composites as an effect of projection, Astronomy and Astrophysics, 371, 267-273, 2001

Rho, J., Petre, R. Mixed-Morphology Supernova Remnants, The Astrophysical Journal, 503, L167-L170, 1998

Shelton, R. L., Cox, D. P., Maciejewski, W., et al. Modeling W44 as a Supernova Remnant in a Density Gradient with a Partially Formed Dense Shell and Thermal Conduction in the Hot Interior. II. The Hydrodynamic Models, The Astrophysical Journal, 524, 192-212, 1999

Slane, P., Smith, R. K., Hughes, J. P., Petre, R. An X-Ray Study of the Supernova Remnant G290.1-0.8, The Astrophysical Journal, 564, 284-290, 2002

Smith, R. K., Brickhouse, N. S., Liedahl, D. A., Raymond, J., C. Collisional Plasma Models with APEC/APED: Emission-Line Diagnostics of Hydrogen-like and Helium-like Ions, The Astrophysical Journal, 556, L91L95, 2001

Smith, R. K., Hughes, J. P., Ionization Equilibrium Timescales in Collisional Plasmas, The Astrophysical Journal, 718, 583-585, 2010

Troja, E., Bocchino, F., Reale, F. XMM-Newton Observations of the Supernova Remnant IC 443. I. Soft X-Ray Emission from Shocked Interstellar Medium, The Astrophysical Journal, 649, 258-267, 2006

Troja, E., Bocchino, F., Miceli, M., Reale, F. XMM-Newton observations of the supernova remnant IC 443. II. Evidence of stellar ejecta in the inner regions, Astronomy and Astrophysics, 485, 777-785, 2008

Uno, S., Mitsuda, K., Inoue, H., et al. X-Ray Spectrum of Supernova 1993J 
Observed with ASCA and Its Evolution 8-572 Days after the Explosion, The Astrophysical Journal, 565, 419-429, 2002

White, R. L., Long, K. S. Supernova remnant evolution in an interstellar medium with evaporating clouds, The Astrophysical Journal, 373, 543-555, 1991

Yamaguchi, H., Koyama, K., Katsudam S., et al. X-Ray Spectroscopy of SN 1006 with Suzaku, Publications of the Astronomical Society of Japan, 60, S141-S152, 2008

Yamaguchi, H., Ozawa, M., Koyama, K., et al. Discovery of Strong Radiative Recombination Continua from the Supernova Remnant IC 443 with Suzaku, The Astrophysical Journal, 705, L6-L9, 2009

Yusef-Zadeh, F., Uchida, K. I., Roberts, D. Shock-Excited OH Maser Emission Outlining the Galactic Center Supernova Remnant G359.1-0.05, Science, 270, 1801-1804, 1995

Zhou, X., Miceli, M., Bocchino, F., et al. Unveiling the spatial structure of the overionized plasma in the supernova remnant W49B, Monthly Notices of the Royal Astronomical Society, 415, 244-250, 2011 